TITLE:

\title{
Physical Activity Mediates the Relationship between Gait Function and Fall Incidence after Total Knee Arthroplasty
}

\section{$\operatorname{AUTHOR(S):~}$}

Taniguchi, Masashi; Sawano, Shinichiro; Maegawa, Shoji; Ikezoe, Tome; Ichihashi, Noriaki

\section{CITATION:}

Taniguchi, Masashi ...[et al]. Physical Activity Mediates the Relationship between Gait Function and Fall Incidence after Total Knee Arthroplasty. The journal of knee surgery 2021, 34(11): 1205-1211

\section{ISSUE DATE:}

2021-09

URL:

http://hdl.handle.net/2433/265248

\section{RIGHT:}

This is the accepted manuscript of the article, which has been published at https://doi.org/10.1055/10.1055/s-00401702165.; The full-text file will be made open to the public on 4 March 2021 in accordance with publisher's 'Terms and Conditions for Self-Archiving'.; This is not the published version. Please cite only the published version.この論文は出版 社版でありません。引用の際には出版社版をご磪認じ利用ください。 


\section{Abstract}

2 The present study aimed to examine (1) the preoperative factors that can predict

3 postoperative falls, (2) whether postoperative physical activity (PA) mediates the

4 relationship between fall incidence and gait function, and (3) whether postoperative PA

5 levels are associated with fall risk in total knee arthroplasty (TKA) patients. Ninety-six

6 patients (mean age; $72.0 \pm 6.1 \mathrm{yrs}$.) who were observed postoperatively for 6 months

7 were selected. Timed-up and go (TUG) was assessed as an indicator of gait function.

8 Fall incidence and PA were investigated for 6 months post-TKA. The body mass index,

9 history of preoperative falls, knee pain, knee extensor strength, range of motion in knee

10 flexion, and modified gait efficacy scale were evaluated. Additionally, postoperative PA

11 levels were categorized into three groups: low: <3000, moderate: $3000-4000$, and high:

$12 \geq 4000$ steps/day. The relative fall incidence rate was calculated according to the total

13 number of falls normalized for every 1000 steps/day for 6 months postoperatively.

14 Twenty-five (26.0\%) of the 96 patients had at least one fall. The TUG, knee pain, and

15 knee extensor strength were identified preoperatively as significant variables affecting

16 postoperative falls. The mediated effects model revealed that postoperative fall

17 incidence was predicted by preoperative TUG and postoperative PA. Postoperative PA was significantly associated with preoperative TUG. Moreover, both the preoperative TUG and postoperative PA were selected as significant variables for predicting fall incidence. Thus, postoperative PA mediates the relationship between gait function and

21 fall incidence after TKA. Furthermore, the relative fall incidence rate associated with a low PA level was significantly higher than that associated with moderate and high PA levels. In conclusion, preoperative assessments of TUG performance, muscle strength, and knee pain were effective in predicting fall risk. Additionally, an increase in PA could 
contribute to reducing fall risk in TKA patients. Therefore, our results suggest that preoperative screening for fall predictors and managing postoperative PA could reduce the fall incidence in TKA patients.

Keywords: Physical activity, fall incidence, gait function and total knee arthroplasty

\section{Text}

\section{Introduction}

The success of total knee arthroplasty (TKA) is measured based on pain relief and improvement of physical function in patients with end-stage knee osteoarthritis (OA). Functional recovery, particularly, improvement of gait function in patients with TKA contributes in enhancing activity of daily living and quality of life. ${ }^{1,2}$ However, the fall incidence was $17 \%-48 \%$ of patients after TKA, which is high compared with asymptomatic healthy older people..$^{3-6}$ A previous study ${ }^{6}$ showed that the first fall occurred in a median of 15 weeks postoperatively, and occurred mostly during walking. Generally, the recovery of physical function reaches a plateau by 6 months after TKA.,8 Therefore, postoperative 6 months is considered important for patients who underwent TKA to recover physical function; nevertheless, fall risk was high during this period due to insufficient functional recovery.

The timed-up and go (TUG) test is useful for screening fall risks in older people with lower function ${ }^{9}$ and reflects the balance performance in patients who underwent TKA. ${ }^{10}$ In previous studies,,${ }^{8,11,12}$ the TUG test was frequently used to assess gait function before and after TKA. Thus, we focused on TUG test that reflects fall risks 
and gait function in patients who underwent TKA. In addition, poor physical function, particularly muscle weakness, ${ }^{5}$ range of motion (ROM) restriction, ${ }^{3}$ and pain $^{13}$ are fall risk factors for patients who underwent TKA. Previous studies ${ }^{14-16}$ reported that the risk of postoperative fall incidence was predicted by a preoperative history of falls, the OA grade of the contralateral knee, and self-efficacy. However, it is not clear whether the preoperative TUG can predict postoperative falls, considering these fall risk factors.

Screening the potential risk factors preoperatively is necessary to reduce fall incidence after TKA.

Increases in physical activity (PA), which targeted approximately 3000 steps per day, was recommended for patients who underwent TKA to enhance gait function during 6 months postoperatively. ${ }^{17}$ Previous studies ${ }^{18,19}$ investigating the relationship between PA levels and fall incidence in community-dwelling older people prospectively showed that high PA level was associated with low fall risk. In contrast, another study ${ }^{20}$ suggested that high PA could increase the exposure to situations during falls. Generally, increases in PA promoted improvement in physical function. ${ }^{21,22}$ In fact, since postoperative PA promoted gait improvement in patients with TKA, ${ }^{17}$ even patients with poor preoperative gait function could reduce the fall incidence risk by increasing postoperative PA. Thus, postoperative PA as a mediator might contribute to improving gait function and reducing the fall risk in patients who underwent TKA. However, to the best of our knowledge, no studies have evaluated whether postoperative PA was a mediator between gait function and fall incidence in patients who underwent TKA. A previous study ${ }^{19}$ has indicated that both high and low PA levels increase the fall incidence risk. ${ }^{23}$ To verify whether fall risk in both low and high PA were higher than 
step counts) to falling. This approach has an advantage when fall risk was not linearly increased with PA. Investigating the relationship between various levels of PA and fall risk could provide valuable information for fall risk by considering PA after TKA. The present study aimed to examine (1) the preoperative factors that can predict postoperative falls, (2) whether postoperative PA mediates the relationship between fall incidence and gait function, and (3) whether postoperative PA levels are associated with fall risk in patients who underwent TKA. We hypothesized that preoperative gait function would predict the postoperative fall incidence; postoperative PA would mediate the relationship between the fall incidence and gait function; and the fall risk would decrease with increasing activity.

\section{Material and Methods}

\section{Study design}

An observational cohort study of patients with knee osteoarthritis patients scheduled for primary TKA was conducted in XXX hospital. Patients who underwent revision TKA, or those who had impairments in the central or peripheral nervous systems, musculoskeletal disorders in other joints, or severe dementia were excluded from the present study. We enrolled 105 patients, and 9 were lost to follow-up; thus, 96 were included for data analysis. For the logistic regression analysis, at least 10 patients per independent variable were required for an adequate sample size. ${ }^{24}$ The logistic regression analysis in this study involved a maximum of 9 independent variables; thus, the sample size was calculated to consist of at least 90 TKA patients. 
of XXX. All patients underwent the same procedure of surgery and rehabilitation. The surgical approach used for TKA was a standard medial parapatellar approach by using a tourniquet. All patients started ambulation using a walker, or a wheelchair if required, on the first postoperative day. Physical therapists provided inhospital rehabilitation for 3 weeks, including passive knee ROM exercise, muscle strength exercise, ambulation exercise, and activity of daily living supervision following the clinical pathway. All patients were followed-up by orthopedic surgeons at postoperative 2, 3, and 6 months and by physical therapists at postoperative 3 and 6 months.

\section{Fall incidence}

Fall incidence was defined as any unintended contact with the ground or floor in accordance with a previous study. ${ }^{25}$ We asked the patients if they had a fall during 6 months preoperatively. During 6 months after TKA, the participants recorded the self-check sheet in case if they had a fall. In addition, physical therapists directly confirmed whether the patients had a fall at 3 and 6 months postoperatively. If the participant had a fall, the physical therapists also confirmed the number of falls and the injury severity.

\section{Patients characteristics, physical function, and self-efficacy}

Patient characteristics, such as age, body mass index, gender, OA status of the contralateral knee (Kellgren/Lawrence grade or received TKA), and the presence of comorbidity (e.g., hypertension, diabetes, lumbar canal stenosis, and chronic respiratory disease), were obtained using clinical records. The TUG test measures the time it takes the patients to stand from a chair, walk a distance of $3 \mathrm{~m}$, turn around, and sit down on a 
chair, as quickly as possible. The participants could use assistive devices during TUG

122

123

124

125

126

127

128

129

130

131

132

133

134

135

136

137

138

139

140

test if needed. The faster trail of the two measurements was used for analysis.

Subjective knee pain during walking was assessed by using a visual analog scale. The knee extensor maximum voluntary contraction strength (knee extensor strength) in the operated side was measured on an isometric dynamometer (Isoforce GT-330; OG

GIKEN Co, Japan) with a knee flexion of $60^{\circ}$. The patients performed knee extensor strength test thrice for $3 \mathrm{~s}$. The maximum value of knee extensor strength was obtained and normalized torque to patient's weight. Passive knee flex ROM was measured by a physical therapist in the supine position by using a goniometer. Moreover, the modified gait efficacy scale (mGES) was used to assess the patient's self-efficacy for the walking task. ${ }^{26}$ The mGES is a 10-term self-report measure that assesses the subjective confidence to safety. Each item was scored individually on a 10-point scale, with 10 indicating the presence of best confidence. The total score is a maximum of 100 points, and a high score indicates higher confidence. Physical therapists assessed physical function and self-efficacy preoperatively and 6 months postoperatively.

\section{Physical activity}

The mean number of steps per day (1000 steps/day) as an index of PA was measured from the time patients were able to walk independently using a cane in the hospital to the postoperative follow-up at 6 months. The patients carried a pedometer with triaxial accelerometer (ES-500; YAMASA, Japan), except for bathing and sleeping, and recorded the number of daily steps in a self-check sheet. In addition, the pedometer which was used in this study was equipped with the memory for 30 days and 35 weeks; thus, we confirmed both the pedometer and self-check sheet. The mean number of steps 
per day for every month and the entire 6 months postoperatively were calculated. PA levels were categorized into three groups (low, moderate, and high) based on the mean number of steps per day 6 months postoperatively. In accordance with a previous study ${ }^{17}$ which recommended patients who underwent TKA to walk approximately 3000 steps/day to improve gait function. The present study defined PA levels as follows: $<3000,3000-4000$, and $\geq 4000$ steps/day as low, moderate, and high PA, respectively. The relative fall incidence rate for the total number of falls normalized for every 1000 steps/day 6 months postoperatively was calculated for each PA level. ${ }^{19}$

\section{Statistical analysis}

The patients were categorized into non-fallers or fallers based on the observational data 6 months postoperatively. The non-paired t-test or Fisher's exact test was performed to compare the two groups (i.e., non-fallers and fallers). Binary logistic regression analysis was applied to predict the fall incidence risk after TKA using preoperative outcome variables and covariates, and the adjusted odds ratio (OR) and 95\% confidence interval (CI) were calculated.

For the mediating effects of PA, we performed binary logistic regression and multiple linear regression in accordance with a previous study ${ }^{27}$ as follows: model 1) dependent variable: presence of fall and independent variable: preoperative TUG; model 2) dependent variable: postoperative PA and independent variable: preoperative TUG; model 3) dependent variable: presence of fall and independent variable: postoperative PA; and model 4) dependent variable: presence of fall and independent variables: preoperative TUG and postoperative PA. First, we confirmed significant associations in models 1-3. Subsequently, as a condition for establishing the indirect effect of PA, we 
examined whether PA was significantly associated with fall in model 4, and simultaneously whether the OR on TUG in model 4 was lower than that in model 1. Subsequently, age, body mass index, presence of preoperative fall, visual analog scale, knee extensor strength, knee flex ROM and mGES as covariates were combined in model 4, and the reduction in OR was calculated (model 5).

Two-way analysis of variance in split-plot design was used to compare the time course of postoperative PA in the two groups. Post hoc analysis was performed using the Tukey test to identify the group difference monthly. One-way analysis of variance was performed to test for possible differences in the relative fall incidence rate between PA levels. Post hoc comparisons were conducted using the Tukey test.

All data were expressed as mean \pm standard deviation. SPSS statistical software (version 22.0; SPSS Japan Inc., Japan) was used for all statistical tests. The significant level was set at $\mathrm{p}<0.05$.

\section{Results}

Of 96 patients, $25(26.0 \%)$ had at least one fall 6 months postoperatively. Of the postoperative fallers, 4 patients fell twice, and one patient fell thrice. Fortunately, there was no fall-related fracture. Table 1 shows demographic characteristics of non-fallers and fallers. The body mass index and preoperative fall incidence in fallers were significantly higher than that in non-fallers, although no significant differences were found between the two groups in terms of age, gender, OA status of the contralateral knee, and the presence of comorbidity. Table 2 shows the results of the physical assessment. The TUG was significantly slower in fallers compared with that in 
non-fallers preoperatively and at 6 months postoperatively. The postoperative mean PA for the fallers during the 6-month follow-up period was significantly lower than that for non-fallers (non-fallers, $4.1 \pm 1.5$ [1000 steps/day]; fallers, $2.7 \pm 0.8$ [1000 steps/day], p $<0.001)$.

Table 3 demonstrates the results of the binary logistic regression models for predicting the fall incidence risk after TKA. The preoperative TUG, VAS, and knee extensor strength were identified as the significant variables affecting postoperative falls.

The results of testing the mediated effect are presented in Fig. 1 and Table 3.

With regard to the results of binary logistic regression in model 1, the preoperative TUG was determined to be a significant fall risk factor. The postoperative PA was significantly associated with the preoperative TUG in model 2 and fall risk factor in model $3(\beta=-0.36,95 \%$ CI: $-0.19--0.06, p<0.001)$. Moreover, both the preoperative TUG and postoperative PA were selected as significant variables for predicting fall risk in model 4. The OR for the TUG decreased from model 1 to model 4, which indicated that the PA mediated the relationship between the preoperative TUG and fall risk after TKA. Similarly, a significant mediated effect was shown in model 5, including covariates.

Fig. 2 shows the time courses of postoperative PA between non-fallers and fallers. Two-way analysis of variance indicated a significant interaction $(\mathrm{F}=3.25, \mathrm{p}=$ $0.029)$ and main effect of time difference $(F=21.12, p<0.001)$ for PA. Post hoc analysis showed that PA in fallers was significantly lower than that in non-fallers throughout 6 months postoperatively. The PA in non-fallers significantly increased in stages throughout postoperative 6 months, whereas it did not increase in fallers. In 
addition, the results of the fall incidence and relative fall incidence rate in each PA level were shown in Table 4. One-way analysis of variance indicated a significant difference in the relative fall incidence rate normalized with daily steps between PA levels ( $\mathrm{F}$ $=4.69, p=0.012)$. The relative fall incidence rate of low PA level was significantly higher than those of moderate and high PA levels, whereas no significant differences were found between moderate and high PA levels.

\section{Discussion}

The result of the present study demonstrated that $26.0 \%$ of patients who underwent TKA had falls 6 months postoperatively. The fall incidence rate in present study was almost equivalent to two previous studies that reported $24.2 \%$ (age; $75.9 \pm$ 5.1 years $)^{4}$ and $32.9 \%$ (age; $75.5 \pm 6.0$ years $)^{3}$; however, it was higher than another study $(17.2 \% \text {, age; } 66.3 \pm 6.6 \text { years })^{6}$. Those differences in fall incidence rates between studies maybe affected by the age the patients underwent TKA. The results of predicting the fall incidence after TKA from preoperative factors revealed that the fall incidence was predicted by the TUG, VAS, and knee extensor strength. The TUG test was useful preoperatively for screening the fall incidence risk in TKA patients, which is consistent with that in previous reports involving older individuals. ${ }^{9}$ In agreement with our hypothesis, these results indicated that postoperative PA mediated the relationship between preoperative gait function and fall incidence after TKA, and moderate and high PA levels were associated with a lower fall risk. To the best of our knowledge, this is the first study that revealed the contribution of postoperative PA to the relationship between gait function and the fall incidence. 

patients with poor TUG performance were at a high risk of falling. In model 3, the results of the multiple linear regression indicated that patients with poor TUG performance were associated with lower PA postoperatively. PA was the predictor of fall incidence risk and had a mediation effect between preoperative gait function and fall incidence after TKA. PA was both the predictor of fall incidence risk and had a mediation effect between gait function and fall incidence, suggesting that an increase in postoperative PA could decrease fall incidence. A previous study ${ }^{17}$ showed that postoperative PA promoted gait function after TKA regardless of low preoperative gait function. The results showed that postoperative PA could contribute to the reduction of fall risk incidence. These relationships were maintained even after covariates were entered. Therefore, these results suggested that the promotion of PA in patients who underwent TKA might be useful to reduce future fall incidence. significantly higher PA compared with fallers, and their PA increased throughout the postoperative 6 months, whereas no significant change was found throughout the postoperative 6 months in fallers. In addition, the relative fall incidence rate in the low PA group was higher than that in the moderate and high PA groups. A previous report ${ }^{20}$ that investigated patients with osteoporotic fractures indicated that high PA increased the relative fall risk. However, our results were consistent with a previous study ${ }^{19}$ for healthy elderly living in the community, and high PA was not associated with fall incidence. Because patients who underwent TKA generally enhanced PA with improvement of postoperative knee function, ${ }^{28}$ continuous monitoring of PA was important. Although previous studies ${ }^{29,30}$ have measured PA during short time periods, 
this present study had a strong point because it continuously investigated the transverse

changes of PA 6 months postoperatively. Thus, these results have suggested that patients

who underwent TKA are recommended to increase postoperative PA in stages and to perform moderate PA, namely >3000 steps/day.

This study provided two major clinical implications for reducing the fall incidence after TKA. First, our results suggest that preoperative assessments of muscle strength, knee pain, as well as TUG performance were effective for predicting the fall risk. Since these factors can be conveniently assessed in the clinical setting, these screening tests may provide valuable information to reduce fall incidence after TKA.

Second, postoperative PA had a mediation effect between gait function and fall incidence, and moderate-to-high PA was associated with a lower fall risk; thus, the monitoring of PA could be important in reducing the fall incidence. Therefore, it is important for the clinician to manage postoperative PA in their patients and provide preoperative screening for fall risk. The present study is an observational study; hence, the aim of future research should be to investigate whether these suggestions can reduce fall incidence after TKA.

This study has some limitations. First, although the fall incidence in patients who underwent TKA is known to be associated with depressive symptoms, they were not investigated in the present study. The mGES could also assess the patient's fear, ${ }^{26}$ and was not related with the fall incidence in the current study. Thus, we expect that the effect of depressive symptoms in the results of this study was not significant. Second, other factors such as surgical factors and medication status were not investigated and may have affected our results. Finally, the survey periods were postoperative 6 months, which could have been longer. Because $48.0 \%$ of the fall incidence occurred 12 months 
289 postoperatively, ${ }^{5}$ future studies are needed to evaluate the fall incidence in the long 290 term.

291

292

293 Conclusion

294 Preoperative assessments of TUG performance, muscle strength, and knee pain were

295 effective for predicting the fall risk. Additionally, postoperative PA mediated the

296 relationship between preoperative gait function and fall incidence after TKA; moderate

297 and high PA levels were associated with a lower fall risk. Therefore, an increase in PA

298 could contribute to reducing the fall risk in patients who underwent TKA. The results of

299 our study suggest that preoperative screening for fall predictors and managing

300 postoperative PA could reduce the fall incidence in TKA patients.

301 


\section{References}

1. George LK, Ruiz D, Jr., Sloan FA. The effects of total knee arthroplasty on physical functioning in the older population. Arthritis Rheum 2008; 58: 3166-3171

2. Naili JE, Wretenberg P, Lindgren V et al. Improved knee biomechanics among patients reporting a good outcome in knee-related quality of life one year after total knee arthroplasty. BMC Musculoskelet Disord 2017; 18: 122

3. Matsumoto H, Okuno M, Nakamura T, Yamamoto K, Hagino H. Fall incidence and risk factors in patients after total knee arthroplasty. Arch Orthop Trauma Surg 2012; 132: 555-563

4. Swinkels A, Newman JH, Allain TJ. A prospective observational study of falling before and after knee replacement surgery. Age Ageing 2009; 38: 175-181

5. Levinger P, Menz HB, Wee E et al. Physiological risk factors for falls in people with knee osteoarthritis before and early after knee replacement surgery. Knee Surg Sports Traumatol Arthrosc 2011; 19: 1082-1089

6. Chan ACM, Jehu DA, Pang MYC. Falls After Total Knee Arthroplasty: Frequency, Circumstances, and Associated Factors-A Prospective Cohort Study. Phys Ther 2018; 98: 767-778

7. Kennedy DM, Stratford PW, Riddle DL, Hanna SE, Gollish JD. Assessing recovery and establishing prognosis following total knee arthroplasty. Phys Ther 2008; 88: 22-32

8. $\quad$ Bade MJ, Kohrt WM, Stevens-Lapsley JE. Outcomes before and after total knee arthroplasty compared to healthy adults. J Orthop Sports Phys Ther 2010; 40: 559-567

9. Schoene D, Wu SM, Mikolaizak AS et al. Discriminative ability and predictive validity of the timed up and go test in identifying older people who fall: systematic review and meta-analysis. J Am Geriatr Soc 2013; 61: 202-208

10. Swinkels A, Allain TJ. Physical performance tests, self-reported outcomes, and accidental falls before and after total knee arthroplasty: an exploratory study. Physiother Theory Pract 2013; 29: 432-442

11. Mizner RL, Petterson SC, Stevens JE, Axe MJ, Snyder-Mackler L. Preoperative quadriceps strength predicts functional ability one 
year after total knee arthroplasty. J Rheumatol 2005; 32: 1533-1539

12. Hiyama Y, Wada O, Nakakita S, Mizuno K. Factors Affecting Mobility after Knee Arthroplasty. Journal of Knee Surgery 2017; 30: 304-308

13. Pelt CE, Anderson AW, Anderson MB, Van Dine C, Peters CL. Postoperative falls after total knee arthroplasty in patients with a femoral nerve catheter: can we reduce the incidence? J Arthroplasty 2014; 29: 1154-1157

14. Riddle DL, Golladay GJ. Preoperative Risk Factors for Postoperative Falls in Persons Undergoing Hip or Knee Arthroplasty: A Longitudinal Study of Data From the Osteoarthritis Initiative. Arch Phys Med Rehabil 2018; 99: 967-972

15. Si HB, Zeng Y, Zhong J et al. The effect of primary total knee arthroplasty on the incidence of falls and balance-related functions in patients with osteoarthritis. Sci Rep 2017; 7: 16583

16. Tsonga T, Michalopoulou M, Kapetanakis S et al. Reduction of Falls and Factors Affecting Falls a Year After Total Knee Arthroplasty in Elderly Patients with Severe Knee Osteoarthritis. Open Orthop J 2016; 10: 522-531

17. Taniguchi M, Sawano S, Kugo M et al. Physical Activity Promotes Gait Improvement in Patients With Total Knee Arthroplasty. J Arthroplasty 2016; 31: 984-988

18. Klenk J, Kerse N, Rapp K et al. Physical Activity and Different Concepts of Fall Risk Estimation in Older People--Results of the ActiFE-Ulm Study. PLoS One 2015; 10: e0129098

19. Jefferis BJ, Merom D, Sartini C et al. Physical Activity and Falls in Older Men: The Critical Role of Mobility Limitations. Med Sci Sports Exerc 2015; 47: 2119-2128

20. Chan BK, Marshall LM, Winters KM et al. Incident fall risk and physical activity and physical performance among older men: the Osteoporotic Fractures in Men Study. Am J Epidemiol 2007; 165: 696-703

21. Brach JS, Simonsick EM, Kritchevsky $\mathrm{S}$ et al. The association between physical function and lifestyle activity and exercise in the health, aging and body composition study. J Am Geriatr Soc 2004; 52: 502-509

22. Ogilvie D, Foster CE, Rothnie $\mathrm{H}$ et al. Interventions to promote walking: systematic review. BMJ 2007; $334: 1204$

23. O'Loughlin JL, Robitaille Y, Boivin JF, Suissa S. Incidence of and risk factors for falls and injurious falls among the 
community-dwelling elderly. Am J Epidemiol 1993; 137: 342-354

24. Peduzzi P, Concato J, Kemper E, Holford TR, Feinstein AR. A simulation study of the number of events per variable in logistic regression analysis. J Clin Epidemiol 1996; 49: 1373-1379

25. Buchner DM, Hornbrook MC, Kutner NG et al. Development of the common data base for the FICSIT trials. J Am Geriatr Soc 1993; 41: 297-308

26. Newell AM, VanSwearingen JM, Hile E, Brach JS. The modified Gait Efficacy Scale: establishing the psychometric properties in older adults. Phys Ther 2012; 92: 318-328

27. Baron RM, Kenny DA. The moderator-mediator variable distinction in social psychological research: conceptual, strategic, and statistical considerations. J Pers Soc Psychol 1986; 51: 1173-1182

28. Tsonga T, Kapetanakis S, Papadopoulos C et al. Evaluation of improvement in quality of life and physical activity after total knee arthroplasty in Greek elderly women. Open Orthop J 2011; 5: 343-347

29. Schmalzried TP, Szuszczewicz ES, Northfield MR et al. Quantitative assessment of walking activity after total hip or knee replacement. J Bone Joint Surg Am 1998; 80: 54-59

30. Tonelli SM, Rakel BA, Cooper NA, Angstom WL, Sluka KA. Women with knee osteoarthritis have more pain and poorer function than men, but similar physical activity prior to total knee replacement. Biol Sex Differ 2011;2: 12 


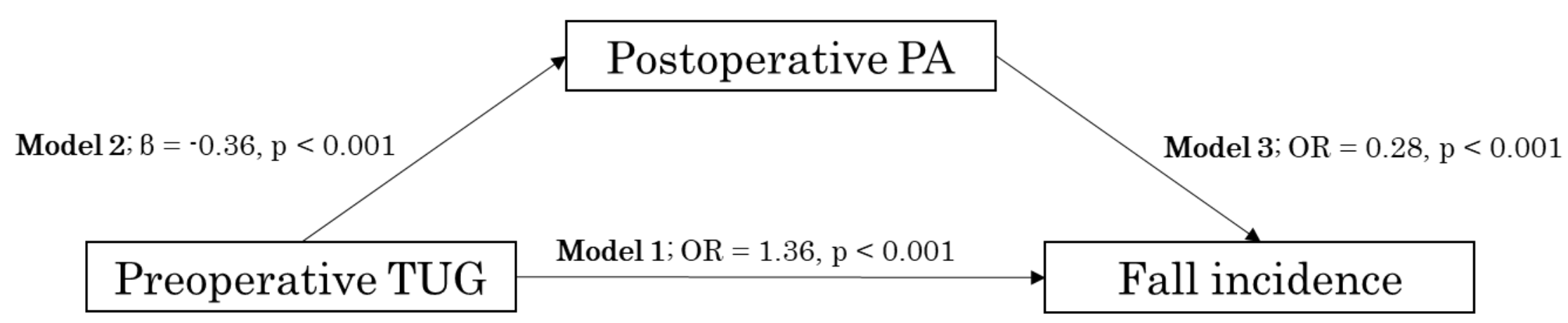

Model 4; OR in preoperative TUG $=1.27, \mathrm{p}=0.003$

OR in postoperative $\mathrm{PA}=0.34, \mathrm{p}=0.004$

Fig. 1 Result of mediating effect of postoperative PA between preoperative TUG and fall incidence after TKA.

Abbreviation: TUG; Timed Up and Go, TKA; Total Knee Arthroplasty, PA; Physical Activity. 


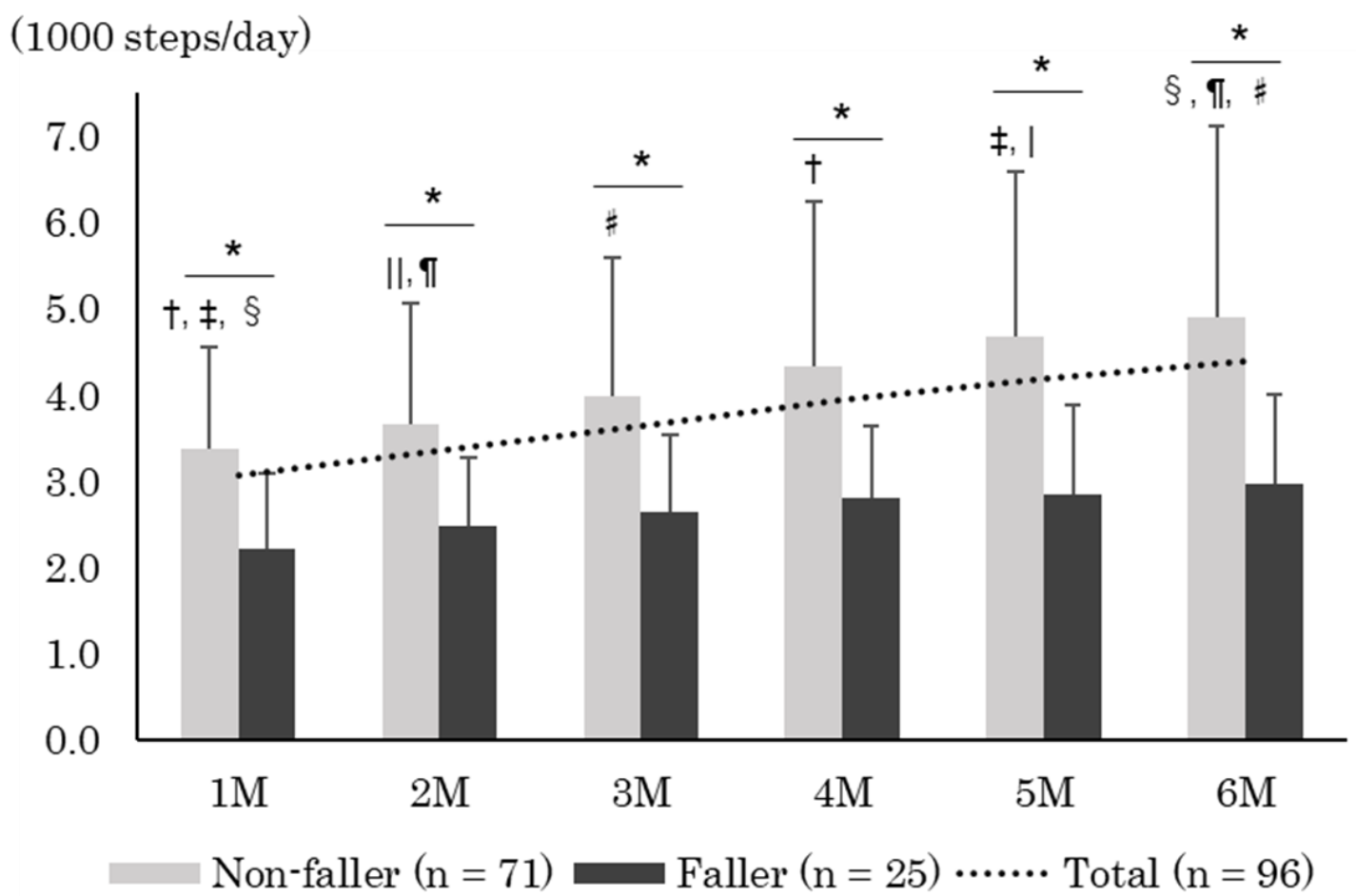

Fig. 2 Time courses of postoperative PA between non-fallers and fallers.

The dotted line shows mean number of steps/day in all patients who underwent TKA (n $=96)$.

* Significant difference between two groups.

${ }^{\dagger}$ Significant time difference in non-fallers between $1 \mathrm{M}$ and $4 \mathrm{M}$, $\ddagger$ between $1 \mathrm{M}$ and $5 \mathrm{M}$, $\S$ between $1 \mathrm{M}$ and $6 \mathrm{M}$, $\|$ between $2 \mathrm{M}$ and $5 \mathrm{M}$, II between $2 \mathrm{M}$ and $6 \mathrm{M}$, and \# between $3 \mathrm{M}$ and $6 \mathrm{M}$, respectively.

Abbreviation: PA; Physical Activity, TKA; Total Knee Arthroplasty. 
Table 1. Demographic characteristics of non-fallers and fallers.

\begin{tabular}{|c|c|c|c|c|c|}
\hline & & & & & \\
\hline & & $(\mathrm{n}=96)$ & $(\mathrm{n}=71)$ & $(\mathrm{n}=25)$ & \\
\hline Age & years & $72.0 \pm 6.1$ & $71.6 \pm 6.2$ & $73.3 \pm 5.9$ & 0.235 \\
\hline Body mass index & $\mathrm{kg} / \mathrm{m}^{2}$ & $26.6 \pm 4.2$ & $26.1 \pm 4.0$ & $28.1 \pm 4.5$ & 0.043 \\
\hline \multirow[t]{2}{*}{ Gender } & women; n (\%) & $88(91.7 \%)$ & $70(90.1 \%)$ & $24(96.0 \%)$ & 0.081 \\
\hline & $\mathrm{K} / \mathrm{L}$ grade $\geq 1$ & $27(28.1 \%)$ & $22(22.9 \%)$ & $5(5.2 \%)$ & \\
\hline \multirow[t]{2}{*}{ OA status of the contralateral knee } & $\mathrm{K} / \mathrm{L}$ grade $\geq 3$ & $41(42.7 \%)$ & $27(28.1 \%)$ & $14(14.6 \%)$ & 0.286 \\
\hline & Received TKA & $28(29.2 \%)$ & $22(22.9 \%)$ & $6(6.3 \%)$ & \\
\hline Hypertension & $\mathrm{n}(\%)$ & $34(35.4 \%)$ & $25(35.2 \%)$ & $9(36.0 \%)$ & 0.944 \\
\hline Diabetes & $\mathrm{n}(\%)$ & $20(20.8 \%)$ & $14(19.7 \%)$ & $6(24.0 \%)$ & 0.652 \\
\hline
\end{tabular}




\begin{tabular}{|c|c|c|c|c|c|}
\hline Lumbar canal stenosis & $\mathrm{n}(\%)$ & $18(18.8 \%)$ & $15(21.1 \%)$ & $3(12.0 \%)$ & 0.317 \\
\hline Chronic respiratory disease & $\mathrm{n}(\%)$ & $8(8.3 \%)$ & $6(6.3 \%)$ & $2(2.1 \%)$ & 1.000 \\
\hline Preoperative fall incidence & $\mathrm{n}(\%)$ & $28(29.2 \%)$ & $16(22.5 \%)$ & $12(48.0 \%)$ & 0.019 \\
\hline
\end{tabular}

Abbreviation: OA; Osteoarthritis, K/L; Kellgren/Lawrence 
Table 2. Physical assessment of non-fallers and fallers at preoperatively and 6 months postoperatively.

\begin{tabular}{|c|c|c|c|c|c|c|c|c|c|}
\hline & & \multicolumn{4}{|c|}{ preoperative } & \multicolumn{4}{|c|}{6 months postoperative } \\
\hline & & $\begin{array}{l}\text { total } \\
(\mathrm{n}=96)\end{array}$ & $\begin{array}{l}\text { Non-fallers } \\
\quad(n=71)\end{array}$ & $\begin{array}{l}\text { Fallers } \\
(\mathrm{n}=25)\end{array}$ & p-value & $\begin{array}{l}\text { total } \\
(n=96)\end{array}$ & $\begin{array}{l}\text { Non-fallers } \\
\quad(n=71)\end{array}$ & $\begin{array}{l}\text { Fallers } \\
(n=25)\end{array}$ & p-value \\
\hline TUG & $\mathrm{s}$ & $11.1 \pm 4.6$ & $9.7 \pm 3.2$ & $14.5 \pm 5.9$ & $<0.001$ & $8.1 \pm 1.9$ & $7.5 \pm 1.1$ & $9.7 \pm 2.6$ & $<0.001$ \\
\hline VAS during gait & $\mathrm{mm}$ & $54.1 \pm 25.6$ & $56.1 \pm 24.5$ & $48.2 \pm 28.3$ & 0.184 & $4.1 \pm 7.8$ & $3.7 \pm 6.3$ & $5.4 \pm 11.1$ & 0.348 \\
\hline $\begin{array}{l}\text { Knee extension } \\
\text { strength }\end{array}$ & $\mathrm{Nm} / \mathrm{kg}$ & $0.8 \pm 0.3$ & $0.9 \pm 0.3$ & $0.6 \pm 0.2$ & $<0.001$ & $1.0 \pm 0.3$ & $1.1 \pm 0.3$ & $0.8 \pm 0.3$ & $<0.001$ \\
\hline Knee flex ROM & deg. & $119.7 \pm 16.4$ & $121.1 \pm 16.3$ & $115.8 \pm 16.5$ & 0.165 & $123.2 \pm 10.8$ & $123.9 \pm 11.3$ & $121.2 \pm 9.1$ & 0.276 \\
\hline mGES & $/ 100$ & $33.0 \pm 18.7$ & $34.5 \pm 18.8$ & $28.5 \pm 18.1$ & 0.167 & $61.8 \pm 21.0$ & $65.1 \pm 20.6$ & $52.5 \pm 19.8$ & 0.009 \\
\hline
\end{tabular}

Abbreviation: TUG; Timed Up and Go, VAS; Visual Analog Scale, ROM; Range of Motion, mGES; modified Gait Efficacy Scale. 
Table 3. Binary logistic regression models for predicting fall incidence after TKA from preoperative factors.

Abbreviation: TKA; Total Knee Arthroplasty.

\begin{tabular}{lccc}
\hline & OR & $95 \%$ CI & $p$-value \\
\hline \hline TUG & 1.28 & $1.07-1.54$ & 0.007 \\
Preoperative fall incidence & 3.07 & $0.74-12.70$ & 0.121 \\
VAS during gait & 0.96 & $0.93-0.99$ & 0.015 \\
Knee extensor strength & 0.02 & $0.01-0.47$ & 0.014 \\
Knee flex ROM & 1.02 & $0.97-1.06$ & 0.464 \\
mGES & 1.00 & $0.96-1.04$ & 0.945 \\
Age & & & \\
Body mass index & 1.01 & $0.90-1.14$ & 0.822 \\
OA status of the contralateral knee & & & 0.797 \\
\hline
\end{tabular}

Abbreviation: OR; Odds Ratio, CI; Confidence Interval, TUG; Timed Up and Go, VAS;

Visual Analog Scale, ROM; Range of Motion, mGES; modified Gait Efficacy Scale; OA; Osteoarthritis. 
Table 4. The mediating effects models of postoperative PA on the relationship between preoperative TUG and fall incidence after TKA.

OR

$95 \% \mathrm{CI}$

$p$-value

Model 1: Univariate

Preoperative TUG

1.36

$1.16-1.59$

$<0.001$

Model 4: Model 1 with mediator

Preoperative TUG

Postoperative PA

Model 5: Model 4 with multiple covariates

Preoperative TUG

Postoperative PA

Preoperative fall incidence

Visual analog scale

Knee extensor strength

Knee flex ROM

mGES

Age
1.27

0.34

$1.01-1.47$

0.036

0.28

$0.11-0.71$

0.007

1.76

$0.37-8.50$

0.481

0.97

$0.93-0.99$

0.030

0.01

$0.00-0.35$

0.011

1.00

$0.96-1.05$

0.910

1.02

$0.97-1.06$

0.464

0.97

$0.86-1.10$

0.673 
Body mass index

OA grade of the contralateral knee
1.02

0.812

$0.32-2.04$

0.658

Abbreviation: OR; Odds Ratio, CI; Confidence Interval, TUG; Timed Up and Go, PA;

Physical Activity, ROM; Range of Motion, mGES; modified Gait Efficacy Scale; OA;

Osteoarthritis. 
Table 5. Fall incidence and incidence rate in each PA level.

\begin{tabular}{|c|c|c|c|c|}
\hline & & low-PA & moderate-PA & high-PA \\
\hline & & $(\mathrm{n}=37)$ & $(\mathrm{n}=27)$ & $(n=32)$ \\
\hline Fall incidence & $\mathrm{n}(\%)$ & $17(45.9 \%)$ & $7(25.9 \%)$ & $1(3.1 \%)$ \\
\hline The relative fall incidence rate & per 1000 steps/d & $0.31 *, \dagger$ & $0.07 *$ & $0.01^{\dagger}$ \\
\hline
\end{tabular}

* Significant group difference between low-and moderate PA; $\mathrm{p}<0.05$.

$\dagger$ Significant group difference between low-and high-PA; $\mathrm{p}<0.05$.

Abbreviation: PA; Physical Activity. 